\section{Mortalidad por olas de calor en la ciudad de Buenos Aires, Argentina (2005-2015)}

\author{
Mortality from heat waves in the city of Buenos \\ Aires, Argentina (2005-2015)
}

\section{Mortalidade por ondas de calor na cidade de Buenos Aires, Argentina (2005-2015)}

Francisco Chesini 1 Rosana Abrutzky 2 Ernesto de Titto 3

doi: 10.1590/0102-311X00165218

\section{Resumen}

El objetivo de este trabajo es analizar la mortalidad durante las olas de calor en la ciudad de Buenos Aires, Argentina, para el período 2005-2015. Utilizamos un diseño de series temporales con modelos aditivos generalizados, vinculando mortalidad con días de ola de calor en todo el período, y con días de la ola de calor de 2013, la más prolongada desde el año 1906, controlando por variables temporales, temperatura media y humedad. Encontramos que el riesgo de muerte por causas naturales se incrementa en un $14 \%(R R=1,140$; IC95\%: 1,108-1,173) durante las olas de calor, respecto al resto de los días del semestre cálido. El incremento se da en ambos sexos y en todos los grupos de edad, siendo más afectados los menores de 15 ( $R R=1,167$; IC95\%: 1,0191,335) y los mayores de 84 años $(R R=1,201$; IC95\%: 1,098-1,313). En la ola de calor de diciembre de 2013 aumentaron 43\% ( $R R=1,428$; IC95\%: 1,399$1,457)$ las muertes diarias totales, valor que sube al 51\% para el grupo de mayores de 84 años $(R R=1,515$; IC95\%: 1,372-1,674) y al 65\% $(R R=1,647$; IC95\%: 1,367-1,986) para las causas renales. Concluimos que las olas de calor constituyen un factor significativo de riesgo de muerte, diferente según sexo y edad, para la población de la ciudad de Buenos Aires.

Mortalidad; Calor; Cambio Climático; Salud Ambiental

\section{Correspondencia}

R. Abrutzky

Instituto de Investigaciones Gino Germani, Facultad de Ciencias Sociales, Universidad de Buenos Aires

Pte. J. E. Uriburu 950, Buenos Aires / Buenos Aires - 1114,

Argentina.

rabrutzky@gmail.com

1 Ministerio de Salud de la Nación, Buenos Aires, Argentina. 2 Facultad de Ciencias Sociales, Universidad de Buenos Aires, Buenos Aires, Argentina.

3 Universidad Isalud, Buenos Aires, Argentina 


\section{Introducción}

Las olas de calor son una amenaza natural para la salud que, debido al cambio climático, han incrementado su frecuencia e intensidad 1,2 . Aunque todavía no hay consenso internacional sobre su definición 3,4,5,6, las distintas definiciones comparten el principio común de "períodos largos de tiempo con temperatura más cálida que la media esperable para un área o región”. En la Argentina, el Servicio Meteorológico Nacional (SMN) define como ola de calor al período en el cual las temperaturas máximas y mínimas igualan o superan, por lo menos durante 3 días consecutivos y en forma simultánea, el percentil 90, calculado a partir de los datos diarios durante los meses de octubre a marzo (semestre cálido en el hemisferio sur) del período 1961-2010 7.

El significativo impacto sanitario de las olas de calor ha sido documentado en numerosos estudios. Por ejemplo, la ocurrida en julio de 1995 en Chicago (EE.UU.), provocó un incremento del 85\% de las muertes (700 casos registrados) 8,9, la del verano de 2003 fue responsable de 14.800 muertes adicionales en Francia y de más de 70.000 en toda Europa 10,11 y la ocurrida en 2010 en la Federación Rusa, con una duración de 44 días, presentó 10.000 muertes en exceso en Moscú 12.

Los adultos mayores, los niños, los enfermos crónicos y las personas que trabajan en ambientes expuestos son los que presentan mayor vulnerabilidad a las olas de calor 1,2,13. Diversos estudios pusieron de manifiesto el incremento en las hospitalizaciones y en la mortalidad por todas las causas durante olas de calor, con incrementos en el riesgo relativo en enfermedades cardiovasculares, cerebrovasculares, respiratorias y renales 5,14,15,16. Es preciso destacar que existe evidencia fisiológica de la relación entre alta temperatura e incrementos de la presión arterial, de la viscosidad de la sangre y de la frecuencia cardíaca por enfermedad cardiovascular, como así también de la ocurrencia de bronco espasmo por enfermedades respiratorias ${ }^{17}$. Además, la capacidad termorreguladora disminuye con la edad, tornando a los adultos mayores más susceptibles a los efectos del calor 18 .

El impacto del calentamiento global en la región es todavía bajo; en la región subtropical de la Argentina la temperatura media anual en superficie tuvo un aumento de temperatura promedio de medio grado entre 1960 y 2010, con calentamientos mayores en sólo algunas zonas e incluso con enfriamientos en otras. Otro efecto que se ha estado observando en toda la región, incluso en el sur y sudeste de Brasil, es la prolongación de las condiciones térmicas del verano en el otoño temprano 19.

Las olas de calor han incremento su frecuencia en el norte y este del país para el mismo período mostrando una pronunciada variabilidad decenal, aunque el mayor número de olas de calor se registró en el período 2001-2010 20. Particularmente, la ola de calor ocurrida en diciembre de 2013 en el centro y norte de Argentina fue probablemente causada por forzantes antropogénicos; observándose que el tiempo de recurrencia de dicho evento sería de 75 años sin forzantes antropogénicos y de 15 años, considerando dichos forzantes 21 .

La Ciudad Autónoma de Buenos Aires es la capital de la República Argentina y el área más densamente poblada del país. Situada a $35^{\circ} \mathrm{S}$ en la orilla oeste del Río de la Plata, es una unidad política de $200 \mathrm{~km}^{2}$, que cuenta con una población de 2.890 .151 habitantes 22 , integrando un área metropolitana de aproximadamente $4.000 \mathrm{~km}^{2}$ con más de 15 millones de habitantes, que desde la perspectiva demográfica presenta dos rasgos distintivos: el envejecimiento poblacional y la feminización 23.

Buenos Aires presenta un clima templado húmedo con veranos cálidos e inviernos frescos e irregulares, con precipitaciones más abundantes en la época estival. La temperatura media anual es de $17,9^{\circ} \mathrm{C}$ y la precipitación anual es de $1.236,3 \mathrm{~mm}$. El SMN ha caracterizado los umbrales de temperatura para las olas de calor en $22^{\circ} \mathrm{C}$ y $32,3^{\circ} \mathrm{C}$ para las temperaturas mínima y máxima, respectivamente.

Buenos Aires ha registrado numerosas olas de calor durante el siglo XX y comienzos del siglo XXI. En la última década (desde el semestre cálido 2004/2005 hasta 2013/2014) se ha registrado al menos un episodio de ola de calor anual, siendo enero el mes con mayor frecuencia de olas de calor, seguido por febrero, y en tercer lugar diciembre 22,24. Cabe señalar que desde el año 2009 el SMN ha implementado en Buenos Aires un sistema de alertas tempranas por olas de calor que podría contribuir a proteger a la población frente a este tipo de eventos 25 .

Escasos trabajos han investigado el impacto sanitario de los cambios de temperatura en Argentina. Así, se ha estudiado la asociación entre condiciones meteorológicas y el número de pacientes atendidos en la sala de emergencia de un hospital privado de Buenos Aires, analizando las consultas por grupo de diagnóstico 26, y la asociación entre los promedios mensuales de las variables meteorológicas 
y la frecuencia mensual de consultas por diversas patologías, a través de las llamadas al Sistema de Atención Médica de Emergencias de Buenos Aires 27. En ambos casos se encontraron algunas asociaciones entre las variables climáticas estudiadas y el perfil de patologías motivo de consulta, aunque pareciera que la relación es más estrecha con la estación del año que con la temperatura. También se analizaron los efectos a corto plazo de los cambios en la temperatura y el monóxido de carbono atmosférico en la mortalidad diaria por todas las causas, y por causas cardiovasculares y respiratorias en Buenos Aires 28, y se investigó la relación entre temperatura y mortalidad general para las ciudades de Rosario y Buenos Aires, encontrando que la relación sigue una forma de "U" con incrementos de muertes en los extremos de temperatura, con preponderancia de las altas temperaturas. En ese trabajo se observó que durante olas de calor ocurridas en Buenos Aires se registraron excesos de mortalidad que van de 20 a $80 \% 29$.

El objetivo del presente trabajo es analizar el impacto de las olas de calor sobre la mortalidad en Buenos Aires durante el período 2005-2015, estimando su peso relativo e identificando el o los grupos poblacionales más vulnerables.

\section{Materiales y métodos}

Para analizar el comportamiento de la mortalidad en Buenos Aires durante una ola de calor se realizó un estudio epidemiológico de tipo ecológico. Se utilizó un diseño de análisis de series temporales con modelos aditivos generalizados (GAM) 30, a fin de evaluar el aumento del riesgo de muerte para los distintos grupos y causas durante el período de ola de calor versus el resto de los días del semestre cálido.

Los datos de mortalidad para los años 2005 a 2015 fueron suministrados por la Dirección de Estadísticas e Información de Salud del Ministerio de Salud de la Nación. Las variables proporcionadas para cada caso de defunción fueron: causa de muerte codificada, según la décima revisión de la Codificación Internacional de Enfermedades (CIE-10), sexo, edad al momento de la muerte y lugar de residencia. Se eliminaron los registros correspondientes a causas externas de muerte (V00 a Z99) 14. Así, se creó una serie para la totalidad de las muertes por causas naturales definidas, ocurridas durante el período, a partir de la cual se generaron sub series con sexo, grupos de edad (seis categorías: 0-14, 15-64, 65-74, 75-84, 85 años y más, y se incluyó un sexto grupo que comprende a todos los mayores de 64 años) y cuatro causas principales de muerte: enfermedades del sistema respiratorio (códigos J00 a J 99), del sistema circulatorio (I00 a I99) y dentro de estas las causas cerebrovasculares (I60 a I69), y enfermedades del sistema genitourinario (N00 a N99).

Los datos meteorológicos fueron provistos por el SMN. Se utilizaron series diarias de temperaturas y humedad relativa, medidas en la Estación Central del SMN en Buenos Aires (Villa Ortúzar, $34^{\circ} 35^{\prime} \mathrm{S}, 58^{\circ} 26^{\prime} \mathrm{O}$ ), y se construyeron dos variables dicotómicas, para la presencia o ausencia de ola de calor y para la presencia o ausencia de ola de calor extendida (la ola de calor de diciembre de 2013 fue la más extensa del registro histórico de la estación Central con una duración de 9 días y es probablemente atribuida al cambio climático 23,24). En el modelo utilizado para evaluar el impacto del resto de las olas del período fueron incluidos los cuatro primeros días de esta ola prolongada. La Figura 1 muestra la evolución de las temperaturas durante el período y los momentos de ola de calor.

De esta manera, se generaron series de 4.018 registros diarios, de los cuales se utilizaron solamente aquellos correspondientes a los meses de octubre, noviembre, diciembre, enero, febrero y marzo, que constituyen el semestre cálido según los parámetros del SMN. Las series finales comprenden datos correspondientes a 1.993 días. A fin de controlar el posible efecto retardado de las olas de calor se generaron rezagos acumulativos de hasta 4 días para las olas cortas y de hasta 8 días para la ola de diciembre 2013. Se controló un posible efecto cosecha para el día siguiente y los tres posteriores a este de la finalización de cada ola.

La Tabla 1 muestra la estadística descriptiva correspondiente a las variables principales del estudio.

Las series y los modelos se trabajaron con el paquete "mgcv" del software R 3.4.2 (http://www.rproject.org), a partir de la fórmula estandarizada:

$$
\log (E(Y))=\beta 0+\beta 1 X 1+\ldots S 1(X 1)+\varepsilon
$$


según la cual Y es el conteo diario de muertes, $\mathrm{E}(\mathrm{Y})$ es el valor esperado para el conteo de mortalidad, $\beta 0$ es el intercepto, $\beta 1$ es el estimador, X1 es la variable concomitante y S1 es la función de suavizamiento. $\beta 1$ expresa el aumento en la mortalidad en presencia de la variable explicativa ola de calor. Los resultados se expresan en términos de razón de riesgo (RR), definida como la diferencia entre casos de expuestos y no expuestos a la ola de calor, con la fórmula:

$$
\mathrm{RR}=\exp (\beta 1)
$$

Figura 1

Evolución de las temperaturas mínima, media y máxima, olas de calor y ola más extensa. Buenos Aires, Argentina, 2005-2015.

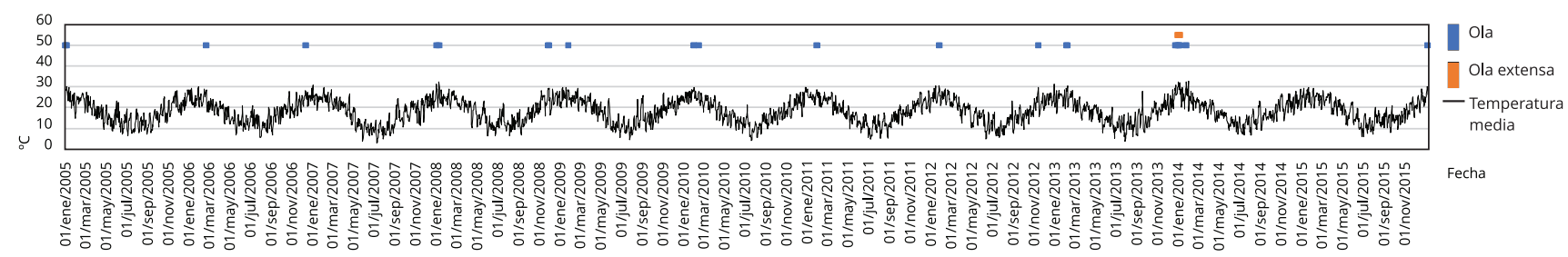

Fuente: elaboración propia en base a datos del Servicio Meteorológico Nacional.

Tabla 1

Estadísticas descriptivas de las variables principales del estudio. Buenos Aires, Argentina, semestres cálidos de 2005 a 2015.

\begin{tabular}{|c|c|c|c|c|c|c|c|}
\hline & Mínimo & 1er cuartil & Mediana & Media & 3er cuartil & Máximo & DE \\
\hline Temperatura máxima $\left({ }^{\circ} \mathrm{C}\right)$ & 13,80 & 24,50 & 27,60 & 27,49 & 30,50 & 39,00 & 4,39 \\
\hline Temperatura media $\left({ }^{\circ} \mathrm{C}\right)$ & 9,50 & 19,70 & 22,50 & 22,28 & 25,10 & 32,70 & 3,88 \\
\hline Temperatura mínima $\left({ }^{\circ} \mathrm{C}\right)$ & 2,50 & 14,70 & 18,10 & 17,56 & 20,60 & 28,20 & 4,09 \\
\hline Humedad relativa (\%) & 29,00 & 59,00 & 68,00 & 67,74 & 75,00 & 98,00 & 11,49 \\
\hline \multicolumn{8}{|l|}{ Mortalidad (casos) } \\
\hline Total & 63,00 & 92,00 & 100,00 & 100,70 & 109,00 & 235,00 & 12,86 \\
\hline Mujeres & 28,00 & 47,00 & 53,00 & 53,56 & 59,00 & 142,00 & 8,87 \\
\hline Varones & 24,00 & 42,00 & 47,00 & 47,14 & 52,00 & 93,00 & 7,47 \\
\hline 0-14 años & 0,00 & 2,00 & 3,00 & 3,67 & 5,00 & 12,00 & 2,03 \\
\hline 15-64 años & 8,00 & 19,00 & 22,00 & 22,57 & 26,00 & 42,00 & 4,82 \\
\hline 65-74 años & 5,00 & 14,00 & 16,00 & 16,81 & 19,00 & 35,00 & 4,32 \\
\hline 75-84 años & 12,00 & 23,00 & 27,00 & 27,63 & 31,00 & 63,00 & 5,90 \\
\hline 85 años y más & 8,00 & 25,00 & 30,00 & 30,02 & 34,00 & 96,00 & 7,09 \\
\hline 65 años y más & 42,00 & 67,00 & 74,00 & 74,46 & 81,00 & 187,00 & 11,27 \\
\hline Causas cardiovasculares & 12,00 & 27,00 & 31,00 & 31,55 & 36,00 & 104,00 & 7,05 \\
\hline Causas respiratorias & 4,00 & 16,00 & 19,00 & 19,34 & 23,00 & 47,00 & 5,24 \\
\hline Causas renales & 0,00 & 3,00 & 5,00 & 4,87 & 6,00 & 14,00 & 2,31 \\
\hline Causas cerebrovasculares & 0,00 & 3,00 & 4,00 & 4,44 & 6,00 & 14,00 & 2,21 \\
\hline
\end{tabular}

DE: desvío estándar.

Fuente: elaboración propia en base a datos del Servicio Meteorológico Nacional y del Ministerio de Salud de la Nación. 
Los modelos se construyeron en dos etapas, en la primera se dio cuenta del comportamiento temporal de las series (mes y día de la semana como variables dicotómicas y suavizamiento por tendencia de largo plazo), y en la segunda etapa se conservaron aquellas variables que mostraron una correlación significativa y se agregaron las variables meteorológicas (temperatura media y humedad) y de ola de calor, con un método de estimación quasi máximo verosimilitud o quasi-Poisson. Este modelo permite aislar el efecto propio de las olas de calor de aquel provocado simplemente por las temperaturas altas.

\section{Resultados}

Durante el período 2005-2015, se registraron 17 olas de calor en la Buenos Aires, con una mediana de duración de 3 días $( \pm 1,8)$. La mayor cantidad de olas se registraron en el mes de enero (7), seguido de diciembre (6) y en tercer lugar noviembre y febrero (2); en los meses de octubre y marzo no se registraron eventos. La Figura 1 muestra la evolución de las temperaturas y las olas de calor durante el período de estudio.

Analizando las muertes acaecidas durante los semestres cálidos del período, se observa que durante las olas de calor hay un incremento significativo en el riesgo de morir por causas naturales $(\mathrm{RR}=1,140$; IC95\%: 1,108-1,173), mientras que la mortalidad atribuida al aumento simple en la temperatura media es de $\mathrm{RR}=1,007$; IC95\%: 1,005-1,009, por cada $1^{\circ} \mathrm{C}$. La Figura 2 muestra los valores de RR para la mortalidad total y por grupos durante las olas de calor y sus respetivos rezagos. No se verificó la presencia de efecto cosecha, es decir, que no se encontró una disminución en el riesgo durante los días posteriores a los eventos; indicando que el aumento de muertes durante las olas de calor no constituye un adelantamiento de decesos inminentes. Por el contrario, tanto las mujeres, los adultos mayores (65 y más años y 85 y más años), como las causas cardiovasculares y respiratorias, alcanzan su riesgo relativo más alto el día posterior a la finalización de la ola de calor.

El riesgo varía levemente según el sexo, siendo $R R=1,081$; IC95\%: 1,034-1,129 para mujeres y $R R=$ 1,074; IC95\%: 1,030-1,121 para los varones. Mientras que en los varones el mayor RR fue durante las olas de calor, para las mujeres el RR más alto (1,092; IC95\%: 1,052-1,134) se alcanza al incluir también el día siguiente a la finalización de la ola, lo que implica que el efecto del calor sobre el organismo afecta a este grupo más allá de la finalización del evento.

En cuanto a los grupos de edad se observaron incrementos estadísticamente significativos en el riesgo de morir en los grupos de 0-14, 15-64, 75-84 y 85 y más, sólo en el grupo de 65-74 no se obser-

\section{Figura 2}

Riesgo relativo (RR) de morir durante olas de calor, según sexo, grupos de edad y causas de defunción. Buenos Aires, Argentina, $2005-2015$.

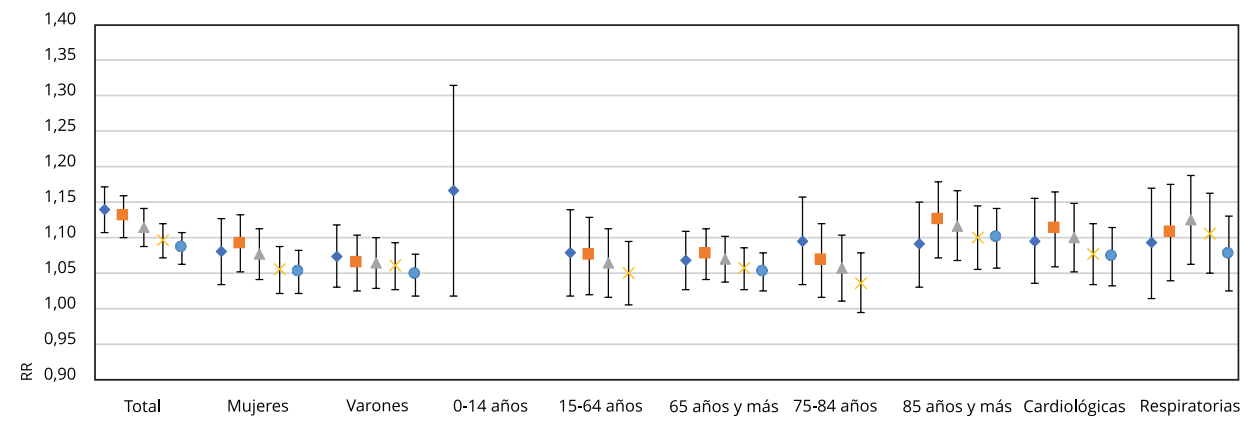

- Ola

- Ola + 1 día

$\Delta \quad$ Ola +2 días

$\times$ Ola +3 días

- Ola +4 días

Nota: se muestran solamente las correlaciones con significancia 0,01 o menor. 
vó un incremento significativo ( $R R=1,024$; IC95\%: 0,957-1,096). En el caso de los niños (0-14 años), quienes presentan el RR más elevado (1,167; IC95\%: 1,019-1,335), el incremento fue significativo sólo al analizar el período de ola de calor, no mostrando significancia al evaluar los efectos desfasados. Los grupos de 15-64 y de 75-84 mostraron significancia estadística al analizar la ola de calor y con la inclusión de los días 1, 2 y 3 posteriores a la ola. Los mayores de 84 años, y el total de los adultos mayores ( 65 y más años), mostraron incrementos significativos en la mortalidad durante la ola y hasta con 4 días de rezago posterior al evento como se observa en la Figura 2. El grupo de 85 y más años presentó el mayor valor de RR no durante la ola, sino al día siguiente de su finalización $(\mathrm{RR}=1,201$; IC95\%: 1,098-1,313).

Las causas de defunción que mostraron un incremento significativo en el riesgo de morir fueron las cardiacas y las respiratorias. La mortalidad por causas cardiovasculares alcanza su RR más alto (1,112; IC95\%: 1,059-1,168), si se incluye el día posterior a la finalización de la ola de calor, y las causas respiratorias lo hacen al segundo día de finalizada la ola $(R R=1,126$; IC95\%: 1,063-1,192). No se observó un aumento significativo en el riesgo de muerte por causas renales, ni cerebrovasculares, durante las olas de calor. Para descartar la posibilidad de efectos diferidos en el tiempo se corrieron, para las causas renales, rezagos de hasta 15 días, no encontrándose correlación significativa en ninguno de ellos.

Finalmente, los cambios en los valores de humedad relativa no modificaron los resultados obtenidos para los grupos y rezagos analizados.

\section{Ola de calor de diciembre de 2013}

En el mes de diciembre de 2013 se produjo la ola de calor más prolongada de los últimos 110 años 24 . Durante nueve días la temperatura máxima media fue de $36,1^{\circ} \mathrm{C}$, y la mínima media de $25,4^{\circ} \mathrm{C}$, siendo los segundos valores más altos alcanzados durante las olas de calor bajo estudio. Asimismo, la temperatura máxima absoluta fue la más alta del período analizado. A esto se suma el hecho de que esta ola ocurrió tres días después de la finalización de otra ola más corta de cuatro días.

El riesgo de morir durante la ola de calor de diciembre de 2013 se incrementó significativamente para la mortalidad general (total de causas naturales), pasando de un 14\% más (en los días de ola de calor), a un 42,8\% más que en cualquier otro día del semestre cálido. Además, el riesgo aumentado se mantiene con la inclusión de los cuatro días posteriores a la finalización de las olas como puede verse en la Figura 3.

En cuanto al comportamiento por sexo, en las mujeres el mayor valor de riesgo relativo se registró incluyendo al día siguiente de la finalización de la ola $(R R=1,395$; IC95\%: 1,286-1,512), mientras que en los varones el pico en el RR se registró al cuarto día ( $R R$ = 1,275; IC95\%: 1,184-1,373) (Figura 4).

\section{Figura 3}

Riesgo relativo (RR) para mortalidad total, olas de calor 2005-2015 vs. ola de calor diciembre 2013. Buenos Aires, Argentina.

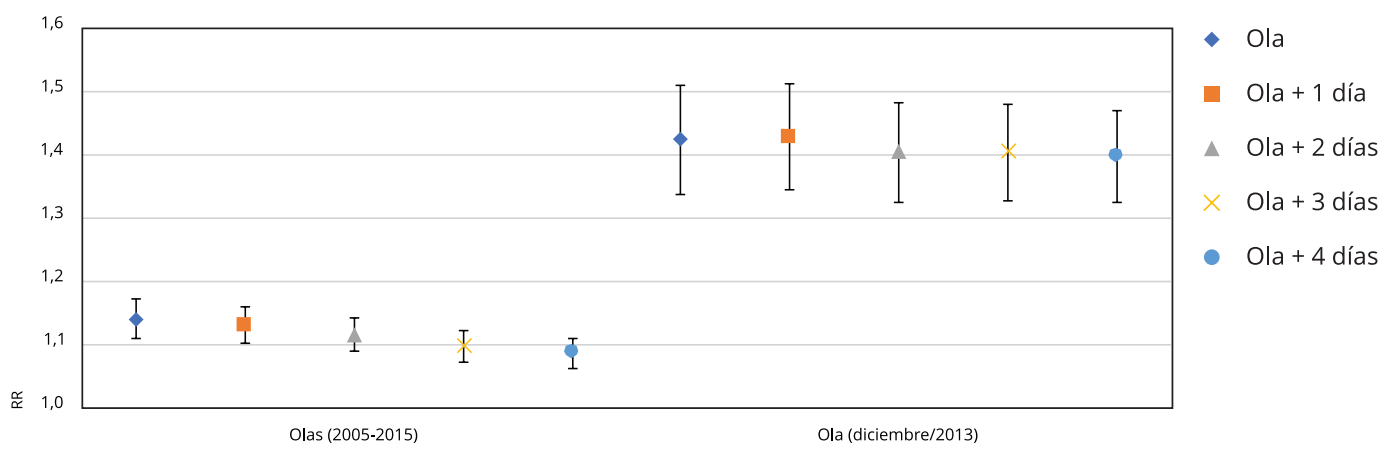




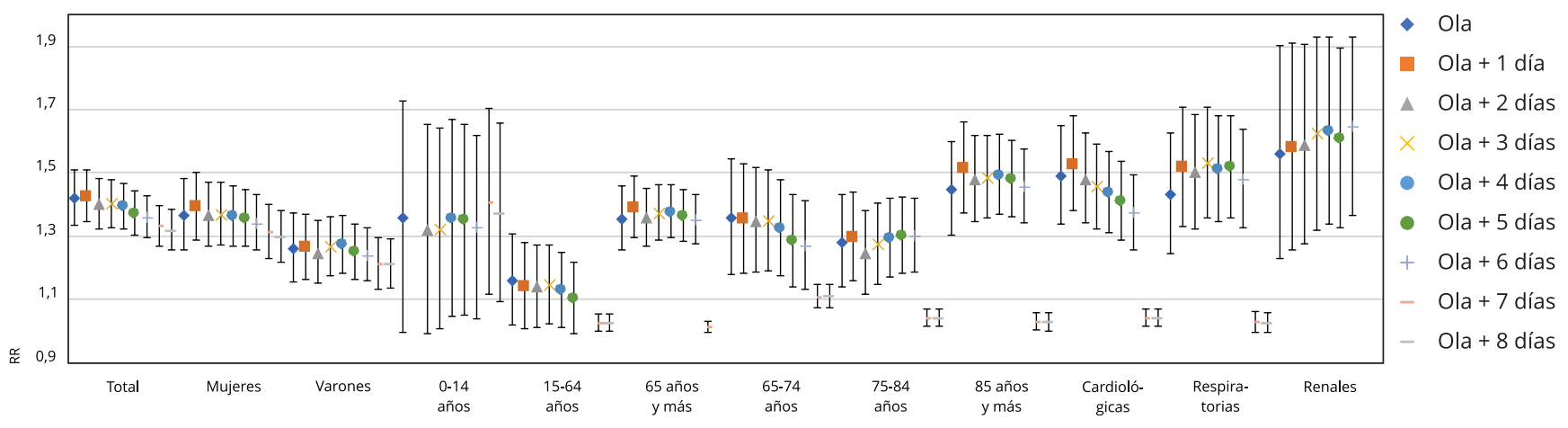

Nota: se muestran solamente las correlaciones con significancia 0,01 o menor.

$\mathrm{Al}$ analizar los incrementos en el riesgo de morir por grupos de edad, todos presentan riesgo relativo incrementado significativamente. Particularmente, el grupo de 0-14 años pierde significancia con el rezago de 1 día, pero la recupera para los rezagos de 2 a 8 días, alcanzando su valor más alto en el día 7, posterior a la finalización de la ola, mientras que el grupo de 15-64 muestra incrementos significativos durante la ola de calor y con rezagos de 1 a 6 días. El mayor incremento en el riesgo de morir lo presentan los adultos de 85 y más años, quienes superan el 50\% de aumento en la mortalidad durante la ola de calor y el día siguiente ( $R R=1,515$; IC95\%: 1,372-1,674).

El grupo de edad de 65-74 que no presentaba incrementos significativos en el riesgo de morir para todas las olas del período 2005-2015 presentó incrementos significativos en la ola de diciembre de 2013 ( RR = 1,361; IC95\%: 1,178-1,572).

En cuanto a las causas de muerte analizadas, excepto el accidente cerebrovascular -que no presentó correlación con esta ola de calor en ninguno de los rezagos analizados- todas ellas presentan un riesgo aumentado que se incrementa posteriormente a la finalización del evento. Las causas de defunción, debidas a problemas renales, no habían presentado sensibilidad a las olas de calor en su conjunto, pero al estudiar un evento extremo, como el de diciembre de 2013, se observó un incremento significativo en el riesgo de morir con pico máximo en el sexto día posterior a la finalización de la ola $(\mathrm{RR}=1,647$; IC95\%: 1,367-1,986), tras el que pierde significancia estadística.

\section{Discusión}

A pesar de la falta de consenso internacional en la definición de una ola de calor, podemos afirmar que Buenos Aires, como muchas otras regiones del planeta, muestra un incremento persistente en la intensidad, frecuencia y/o duración de este tipo de eventos 22.

Las olas de calor analizadas presentan diferente duración entre sí, y distinta intensidad (temperaturas máximas y mínimas). Sin embargo, el evento definido -con criterios climatológicos- por el SMN como "ola de calor" constituye un predictor de un incremento en el riesgo de muerte para la población total y por grupos.

Resulta interesante la comparación entre los resultados obtenidos para la ola de diciembre de 2013, versus las restantes 16 olas de calor del período 2005-2015, dado que dicha ola de calor extrema fue atribuida a los forzantes antropogénicos y se espera que eventos de estas características sean más frecuentes, debido al cambio climático 23 . La persistencia de altas temperaturas durante nueve días mostró un impacto aumentado, tanto para la mortalidad total, como para todos los grupos analizados, incluyendo el de adultos entre 65 y 74 años, que no presentó riesgo aumentado durante las olas cortas. 
Asimismo, durante la ola de diciembre de 2013 se incrementó la diferencia en el riesgo según sexo, pasando de 0,7\% más riesgo para las mujeres durante todas las olas a 12\% en la ola más extrema. Además, se observó un efecto sobre la mortalidad por causas renales que no aparecía con posterioridad a las olas cortas (10 de las 17 olas ocurridas en el período tuvieron una duración de tres días). Esto implicaría la necesidad de un seguimiento específico para aquella población con vulnerabilidad renal ante eventos de estas características.

No es extraño que el impacto sanitario de una ola larga, como la de 2013, sea más importante que el de las olas cortas: en una ola larga la temperatura nocturna se mantiene elevada, impidiendo la recuperación. A ello debe sumarse el impacto del efecto "isla de calor", la disminución de la evaporación, la reducida cobertura vegetal y la impermeabilización superficial por el desarrollo urbano 16,31,32. Este fenómeno ha sido estudiado para Buenos Aires, encontrando que las mayores diferencias de temperatura entre la ciudad y su entorno rural se registran durante las noches de verano 33 . Es también razonable considerar que la duración de la ola puede haber incrementado el riesgo para poblaciones que durante fenómenos más cortos no mostraban vulnerabilidad.

Un factor a considerar en la ola larga de 2013 es que esta ocurrió tempranamente, en el semestre cálido, lo cual posiblemente haya dificultado la aclimatación de la población expuesta, tal como sugieren otros autores 34,35 .

Aunque estudios anteriores 14 encuentran una vinculación entre las olas de calor y el accidente cerebrovascular, como causa de muerte, en nuestros datos dicha correlación no se verifica. Esto, por sí solo, no implica la ausencia de relación, ya que podría deberse a una mala clasificación de la causa de defunción en los registros, o al hecho de que por tratarse de una causa minoritaria la correlación exista, pero no sea estadísticamente significativa.

Debe destacarse la dificultad para comparar los resultados encontrados con los presentados por otros autores en diversos países por la falta de consenso en la definición de una ola de calor.

Por el tipo de datos utilizado no podemos conocer, excepto como grandes grupos demográficos, quiénes son los individuos más afectados por las altas temperaturas. Por otro lado, las admisiones hospitalarias, generalmente, se deben al agravamiento de condiciones sanitarias preexistentes más que al calor propiamente dicho ${ }^{4}$, por lo que se requieren nuevos estudios que analicen el impacto en pacientes con enfermedades preexistentes y la vulnerabilidad de diferentes sectores de la población, según situación socioeconómica y lugar de la vivienda, y el trabajo para orientar acciones concretas de protección de los grupos más vulnerables.

Si bien el presente estudio presenta las limitaciones propias de un diseño ecológico, que no puede establecer la causa de la correlación entre uno y otro evento (ola de calor y muerte), los mecanismos biológicos implicados permiten afirmar que la persistencia de temperaturas elevadas durante varios días durante el semestre cálido provoca o facilita el aumento de decesos en la Buenos Aires, como se ha demostrado en otras ciudades 5,32 .

Una limitación de este estudio fue la imposibilidad de controlar el factor de confusión de los contaminantes atmosféricos (ozono, material particulado, monóxido de carbono y óxidos sulfúricos y nitrosos), debido a no contar con series completas para el período analizado. Sin embargo, trabajos previos sugieren que este factor de confusión tiene un impacto moderado, si tiene alguno 5,36.

Para dimensionar el impacto sanitario de las olas de calor, en términos de mortalidad, cabe recordar que Buenos Aires tiene desde hace más de 30 años una población estabilizada en el entorno de los tres millones de habitantes. Aproximadamente el 1\% de ellos fallece anualmente (unos 32.500, el 99\% por causas definidas de las cuales las causas externas dan cuenta del 3\%), dejando un promedio de 89 muertes diarias (85 por causas naturales). Las olas de calor agregan a esta cifra 11 muertes diarias. Queda por investigar el impacto en la morbilidad.

\section{Conclusión}

Los resultados obtenidos permiten concluir que las olas de calor constituyen un factor de riesgo de muerte, diferente según sexo y edad, para la población de la ciudad de Buenos Aires. Una ola de calor prolongada, como la ocurrida durante 2013, produce un impacto mucho mayor en cuanto al riesgo de muerte. 
Estos resultados deberían ser considerados para mejorar el sistema de alertas tempranas que existe en la región, y para la elaboración de nuevas medidas de promoción de la salud, a nivel individual y comunitario, a fin de adaptarse a un escenario futuro con olas de calor más frecuentes e intensas y para procurar así reducir su impacto sanitario.

\section{Colaboradores}

F. Chesini, R. Abrutzky y E. de Titto contribuyeron con la concepción y diseño del trabajo, análisis e interpretación de los resultados, redacción del manuscrito y aprobación de la versión final a ser publicada.

\section{Informaciones adicionales}

ORCID: Francisco Chesini (0000-0001-6555458X); Rosana Abrutzky (0000-0002-5580-0211); Ernesto de Titto (0000-0002-4375-3278).

\section{Agradecimientos}

Los autores agradecen a la Dirección de Estadísticas e Información en Salud del Ministerio de Salud de la Nación y al Servicio Meteorológico Nacional por la provisión de los datos de mortalidad y de variables meteorológicas respectivamente.

\section{Referencias}

1. McGregor GR, Bessemoulin P, Ebi K, Menne $B$. Heatwaves and health: guidance on warning-system development. Geneva: World Meteorological Organization/World Health Organization; 2015.

2. Smith KR, Woodward A, Campbell-Lendrum D, Chadee DD, Honda Y, Liu Q, et al. Human health: impacts, adaptation, and co-benefits. In: Field CB, Barros VR, Dokken DJ, Mach KJ, Mastrandrea MD, Bilir TE, et al., editors. Climate change 2014: impacts, adaptation, and vulnerability. Cambridge/New York: Cambridge University Press; 2014. p. 709-54.

3. Montero JC, Mirón IJ, Criado JJ, Linares C, Díaz J. Difficulties of defining the term, "heat wave”, in public health. Int J Environ Health Res 2013; 23:377-9.

4. Perkins SE. A review on the scientific understanding of heatwaves: their measurement, driving mechanisms, and changes at the global scale. Atmos Res 2015; 164/165:242-67.

5. Son JY, Gouveia N, Bravo MA, de Freitas CU, Bell ML. The impact of temperature on mortality in a subtropical city: effects of cold, heat, and heat waves in São Paulo, Brazil. Int J Biometeorol 2016; 60:113-21.

6. Guo Y, Gasparrini A, Armstrong BG, Tawatsupa B, Tobias A, Lavigne E, et al. Temperature variability and mortality: a multi-country study. Environ Health Perspect 2016; 124:1554-9.

7. Servicio Meteorológico Nacional. Olas de calor. http://www3.smn.gob.ar/serviciosclima ticos/? mod=elclima\&id=13 (accedido el 19/ Mar/2019).

8. Whitman S, Good G, Donoghue ER, Benbow N, Shou W, Mou S. Mortality in Chicago attributed to the July 1995 heat wave. Am J Public Health 1997; 87:1515-8.

9. Centers for Disease Control and Prevention. Heat-related mortality - Chicago, July 1995. MMWR Morb Mortal Wkly Rep 1995; 44:577-9.

10. Vandentorren S, Suzan F, Medina S, Pascal M, Maulpoix A, Cohen JC, et al. Mortality in 13 French cities during the August 2003 heat wave. Am J Public Health 2004; 94:1518-20.

11. Robine JM, Cheung SL, Le Roy S, Van Oyen $\mathrm{H}$, Griffiths C, Michel JP, et al. Death toll exceeded 70,000 in Europe during the summer of 2003. C R Biol 2008; 331:171-8. 
12. Shaposhnikov D, Revich B, Bellander T, Bero Bedada G, Bottai M, Kharkova T, et al. Mortality related to air pollution with the Moscow heat wave and wildfire of 2010. Epidemiology 2014; 25:359-64.

13. McGeehin MA, Mirabelli M. The potential impacts of climate variability and change on temperature-related morbidity and mortality in the United States. Environ Health Perspect 2001; 109:185-9.

14. D’Ippoliti D, Michelozzi P, Marino C, de'Donato F, Menne B, Katsouyanni K, et al. The impact of heat waves on mortality in 9 european cities: results from the EuroHEAT project. Environ Health 2010; 9:37.

15. Gronlund CJ, Zanobetti A, Schwartz JD, Wellenius GA, O'Neill MS. Heat, heat waves, and hospital admissions among the elderly in the United States, 1992-2006. Environ Health Perspect 2014; 122:1187-92.

16. Kovats RS, Hajat S. Heat stress and public health: a critical review. Annu Rev Public Health 2008; 29:41-55.

17. Campbell-Lendrum D, Woodruff R. Climate change quantifing the health impacts in national and local levels. Geneva: World Health Organization; 2007.

18. Martínez-Navarro F, Simón-Soria F, LópezAbente G. Valoración del impacto de la ola de calor del verano de 2003 sobre la mortalidad. Gac Sanit 2004; 18:250-8.

19. Barros V, Camilloni I. La Argentina y el cambio climático. De la física a la política. Buenos Aires: Editorial Eudeba; 2016.

20. Rusticucci M, Kyselý J, Almeira G, Lhotka O. Long-term variability of heat waves in Argentina and recurrence probability of the severe 2008 heat wave in Buenos Aires. Theor Appl Climatol 2016; 124:679-89.

21. Hannart A, Vera C, Otto FE, Cerne B. Causal influence of anthropogenic forcings on the Argentinian heat wave of December 2013. Special Suppl Bull Am Meteorol Soc 2015; 96:541-5.

22. Instituto Nacional de Estadística y Censos. Cuadro P5-P. Ciudad Autónoma de Buenos Aires. Población total por país de nacimiento, según sexo y grupo de edad. Año 2010. https:// www.indec.gob.ar/censos_provinciales. asp?id_tema_1 $=2 \&$ id_tema_2 $=41 \&$ id_tema_3 $=135 \& \mathrm{p}=02 \& \mathrm{~d}=999 \& \mathrm{t}=3 \& \mathrm{~s}=0 \& \mathrm{c}=2010$ (accedido el 04/Abr/2018).

23. Gobierno de la Ciudad de Buenos Aires. Ciudad de Buenos Aires. http://www.buenosai res.gob.ar/laciudad/ciudad (accedido el 12/ Abr/2018).

24. Servicio Meteorológico Nacional. Resumen estadístico y cronológico de las olas de calor en la Ciudad de Buenos Aires (actualización diciembre 2016). http://www3.smn.gob.ar/ser viciosclimaticos/clima/archivo/OlasdeCalor_ BuenosAires.pdf (accedido el 19/Mar/2018).
25. Suaya M, Almeira G. Alertas meteorológicas y de olas de calor sobre la Ciudad Autónoma de Buenos Aires: descripción y verificación. In: XI Congreso Argentino de Meteorología. http:// www.congremet.prmarg.org/upload/suayamartina2.pdf.

26. Rusticucci M, Bettolli ML, Harris MA. Association between weather conditions and the number of patients at the emergency room in an Argentine hospital. Int J Biometeorol 2002; 46:42-51.

27. Alexander P. Association of monthly frequencies of diverse diseases in the calls to the public emergency service of the city of Buenos Aires during 1999-2004 with meteorological variables and seasons. Int J Biometeorol 2013; 57:83-90.

28. Abrutzky R, Dawidowski L, Matus P, Romero-Lankao P. Health effects on climate and air pollution in Buenos Aires: a first series analysis. J Environ Prot (Irvine, Calif) 2012; 3:26271.

29. Almeira G, Rusticucci M, Suaya M. Relación entre mortalidad y temperaturas extremas en Buenos Aires y Rosario. Meteorológica 2016; 41:65-79.

30. Hastie TJ, Tibshirani RJ. Generalized additive models. Boca Ratón: Chapman \& Hall/CRC; 1990.

31. Patz JA, Campbell-Lendrum D, Holloway T, Foley JA. Impact of regional climate change on human health. Nature 2005; 438:310-7.

32. Murage P, Hajat S, Kovats RS. Effect of nighttime temperatures on cause and age-specific mortality in London. Environ Epidemiol 2017; 1:e005.

33. Camilloni I, Barrucand M. Temporal variability of the Buenos Aires, Argentina, urban heat island. Theor Appl Climatol 2012; 107:47-58.

34. Curriero FC, Heiner KS, Samet JM, Zeger SL, Patz JA. Temperature mortality in 11 cities of the Eastern of the United States. Am J Epidemiol 2002; 155:80-7.

35. Weisskopf MG, Anderson HA, Foldy S, Hanrahan LP, Blair K, Torok TJ, et al. Heat wave morbidity and mortality, Milwaukee, Wis. 1999 vs 1995: an improved response? Am J Public Health 2002; 92:830-3.

36. Buckley JP, Samet JM, Richardson DB. Does air pollution confound studies of temperature. Epidemiology 2014; 25:242-5. 


\section{Abstract}

This study aimed to analyze mortality during heat waves in the city of Buenos Aires, Argentina, in 2005-2015. We used a time series design with generalized additive models, linking mortality to days of heat waves throughout the period, and to days of the heat wave in 2013, the longest wave since 1906, controlling for time variables, mean temperature, and humidity. Risk of death from natural causes increased by $14 \%(R R=1.140 ; 95 \%$ CI: 1.108-1.173) during heat waves when compared to the other days in the hot season. The increase occurred in both sexes and in all age groups, and individuals under 15 years of age were more affected $(R R=1.167 ; 95 \% C I: 1.019-1.335)$ as were those over 84 years $(R R=1.201$; 95\%CI: $1.098-1.313)$. The heat wave in December 2013 showed an increase of $43 \%(R R=1.428$; 95\%CI: $1.399-1.457)$ in total daily deaths, increasing to $51 \%$ in individuals over 84 years $(R R=1.515$; 95\%CI: 1.372 $1.674)$ and $65 \%(R R=1.647 ; 95 \% C I: 1.367-1.986)$ for renal causes. We conclude that heat waves pose a significant risk of death, differing according to sex and age in the population of Buenos Aires.

Mortality; Hot Temperature; Climate Change; Environmental Health

\section{Resumo}

O objetivo deste trabalho é analisar a mortalidade durante as ondas de calor na cidade de Buenos Aires, Argentina, no período de 2005-2015. Foi usado um desenho de séries temporais com modelos aditivos generalizados, vinculando a mortalidade com dias de onda de calor em todo o período, e com dias da onda de calor em 2013, a mais prolongada desde o ano de 1906, controlando através de variáveis temporais, temperatura media e umidade. Encontramos que o risco de morte por causas naturais teve um incremento de $14 \%(R R=1,140$; IC95\%: $1,108-1,173)$ durante as ondas de calor, ao respeito do resto dos dias do semestre cálido. O incremento se produz em ambos os sexos e em todos os grupos de idade, sendo mais afetados os menores de $15(R R=1,167$; IC95\%: 1,019-1,335) e os maiores de 84 anos ( $R R=1,201$; IC95\%: 1,098-1,313). Na onda de calor que ocorreu em dezembro de 2013 aumentaram um 43\% ( $R R=1,428$; IC95\%: 1,399$1,457)$ as mortes diárias totais, valor que sobe a 51\% para o grupo de pessoas maiores de 84 anos $(R R=1,515$; IC95\%: 1,372-1,674) e a 65\% (RR = 1,647; IC95\%: 1,367-1,986) para as causas renais, Concluimos que as ondas de calor constituem um fator significativo de risco de morte, diferente segundo o sexo e idade, para a população da cidade de Buenos Aires.

Mortalidade; Temperatura Alta; Mudança

Climática; Saúde Ambiental
Recibido el 22/Ago/2018

Versión final presentada el 08/Abr/2019

Aprobado el 15/Abr/2019 